\section{D) Check for updates}

Cite this: Food Funct., 2017, 8, 4129

\title{
The effect of cognitive demand on performance of an executive function task following wild blueberry supplementation in 7 to 10 years old children $\uparrow$
}

\begin{abstract}
A. R. Whyte, (D) G. Schafer (D) and C. M. Williams (D) *
The cognitive benefits of acute flavonoid interventions have been well documented, however, research to date has found that, depending on developmental stage, these benefits manifest themselves in different cognitive domains. It is argued that the lack of global cognitive effects following flavonoid intervention may be a result of insufficient task sensitivity for those domains where no benefits are found. In children, executive function is a cognitive domain which has shown little apparent benefit following flavonoid intervention. Here, we describe a Modified Attention Network Task (MANT) designed to vary levels of cognitive demand across trials in order to investigate whether flavonoid related benefits can be shown for executive function when task sensitivity is carefully manipulated. Twenty-one children were recruited to a double blind cross-over study consuming $30 \mathrm{~g}$ freeze dried blueberry powder (WBB) or placebo before being tested at 3 hours. Performance in the WBB condition was found to be significantly faster in comparison to placebo particularly on more cognitively demanding incongruent and high load trials. Trials in which a visual cue alerted participants to the imminent appearance of the target also showed better performance following WBB administration. We conclude that WBB administration can enhance executive function during demanding elements of a task, but that the complexity and demand of the task as a whole may be equally important to performance.
\end{abstract}

Received 8th June 2017, Accepted 29th August 2017 DOI: $10.1039 / \mathrm{c} 7 \mathrm{fo} 00832 \mathrm{e}$ rsc.li/food-function reported by Bell et al., ${ }^{3}$ there are indications that different cognitive domains are sensitive to flavonoid interventions at different ages. For example, episodic memory benefits have been found in children and aging adults ${ }^{18,21,22}$ but not young adults, whilst improvements in executive functioning have been robustly produced in young adults ${ }^{12-14,16}$ but findings have been less convincing in children and older adults. Whilst these different effects may reflect genuine lifespan changes, given the relatively small number of studies which have investigated the effects of flavonoids on those at critical developmental stages, an equally likely possibility is that the tasks used were not sufficiently sensitive to find effects regardless of cognitive domain. Indeed, neuroimaging studies indicate differences in brain activation following flavonoid interventions even when clear behavioural differences have not been found. ${ }^{17,23-25}$ Furthermore, Scholey et al. ${ }^{16}$ found cocoa flavanol related improved executive function effects in young adults when employing a sustained mental effort battery designed to reduce ceiling effects, and, in a chronic anthocyanin rich blueberry intervention with older adults, Miller et $a l .{ }^{26}$ noted that, from a wide battery of cognitive tests treatment related improvements were found on the more challenging executive function tasks.
School of Psychology and Clinical Language Sciences, University of Reading, Earley Gate, Whiteknights, Reading RG6 6AL, UK. E-mail: claire.williams@reading.ac.uk $\dagger$ Electronic supplementary information (ESI) available. See DOI: 10.1039/ c7fo00832e 
Recent evidence from our laboratory indicates the benefits of flavonoid supplementation may be modulated by the level of cognitive demand associated with a task. ${ }^{21}$ Here, $7-10$ years old children were supplemented with an anthocyanin-rich wild blueberry (WBB) intervention before completing a Flanker task. Three hours after treatment with an intervention containing $253 \mathrm{mg}$ anthocyanins, children showed significantly greater accuracy on cognitively demanding incongruent trials, while no such effect was found for the less demanding congruent trials.

We hypothesise that as the cognitive difficulty or demand of a task increases, individuals will initially show a similar reduction in performance regardless of whether they have been supplemented with a flavonoid or vehicle intervention. However, beyond a certain point, as cognitive difficulty continues to increase, the performance of individuals supplemented with placebo will fall off more rapidly than those supplemented with a flavonoid intervention. If this model is correct, flavonoid interventions will produce advantages in performance when the task is sufficiently demanding to negatively affect the performance, but not so demanding that it is impossible to perform. Successful flavonoid intervention should thus manifest itself through sustained performance on the task beyond what would be achievable with a placebo intervention.

Here we describe a modified attention network $\operatorname{task}^{27}$ designed to dynamically manipulate the level of demand on visual load, stimulus duration, response interference, orientation, and alerting. In the original Attention Network Task, participants responded to a central arrow in an array of arrows which could be facing in a congruent $(e . g .<<<<)$ or an incongruent (e.g. $<<><<)$ direction. Cueing information alerting the participant to the onset and location of the arrow were also employed. Here we further manipulate task demands by manipulating the number of flanking arrows, the length of time the target appeared on screen, and the level of background noise.

Using the same experimental conditions as we have previously described ${ }^{21}$ with our WBB intervention containing $253 \mathrm{mg}$ anthocyanins and testing occurring 3 hours post consumption, we hypothesise that WBB-treated participants will perform more effectively in comparison to placebo intervention on the more cognitively demanding trials of this task. For example, participants would be expected to respond more accurately and rapidly to un-cued trials with high visual load, incongruent flanking arrows, $500 \mathrm{~ms}$ stimulus duration, and distracting background noise following WBB treatment in comparison to vehicle. However, for less cognitively demanding trials, such as cued trials with low visual load, congruent flanking arrows, $120 \mathrm{~ms}$ stimulus duration, and no distracting noise we would predict no difference between treatments. Between these two extremes, consideration of trials where the cognitive demand of the individual variables is mixed, should allow us to more closely identify the areas and points at which the benefits of flavonoid intervention begin to be seen.

\section{Methods}

This study was reviewed by the University of Reading Research Ethics Committee and was given a favourable ethical opinion for conduct.

\section{Participants}

Based on the results from Whyte et al. $G^{*}$ Power analysis software was used to conduct an a priori analysis using an effect size of 0.45 , and alpha level of 0.05 . The analysis indicated that 21 participants would be required to achieve a power of 0.8 . Twenty one children (10 female) aged between 7 and 10 were recruited from schools located within ABC1 (primarily middle class) areas local to Reading University.

Schools were contacted directly by the researcher and, following permission to proceed, a brief talk at the school was given to invite children of the relevant age group to participate. All children were also given an information pack containing a child appropriate written invitation and further invitation and information sheets for the parent/legal guardian. Parents/legal guardians were asked to indicate their interest in taking part in the study by return of mail. Appropriate consent forms were completed by both the child and parent/legal guardian before testing commenced.

On initial recruitment, parents/legal guardians confirmed that participants spoke English as a first language, had not been diagnosed with ADHD or dyslexia, and had no known intolerance to any fruit or fruit juice. Participants completed the Raven's Coloured Progressive Matrices ${ }^{28}$ as a measure of fluid intelligence. Parents/legal guardians also completed the ADHD rating scale IV, ${ }^{29}$ the Edinburgh handedness inventory ${ }^{30}$ and confirmed average daily fruit and vegetable consumption on behalf of the child. Demographic results from these measures are shown in Table 1.

\section{Materials}

Anthocyanin content of the blueberry powder was analysed as detailed in Rodriguez et al. ${ }^{31}$ The $253 \mathrm{mg}$ blueberry anthocyanin treatment contained $30 \mathrm{~g}$ freeze dried wild blueberry powder $(108 \mathrm{kcal})$ while the placebo treatment contained $8.9 \mathrm{~g}$ of fructose, $7.99 \mathrm{~g}$ of glucose and $4 \mathrm{mg}$ of vitamin $\mathrm{C}$ matching the levels of these nutrients found in our active treatment.

Table 1 Demographic results for participants combined and by gender, $\mathrm{SD}$ in parenthesis ( $N=$ number of participants, RCPM = Rey's coloured progressive matrices, $\mathrm{ADHD}=$ attention deficit hyperactivity disorder, $\mathrm{F} \& \mathrm{~V}=$ fruit and vegetables)

\begin{tabular}{llll}
\hline Variable & All participants & Females & Males \\
\hline$N$ & 21 & 10 & 11 \\
Age & 8.11 years $(0.62)$ & 8.11 years $(0.46)$ & 9.0 years $(0.52)$ \\
RCPM & $27.6(4.28)$ & $26.6(5.1)$ & $28.5(3.36)$ \\
RCPM \%ile & $59.8(23.2)$ & $55(28.5)$ & $64.9(17.4)$ \\
ADHD IV & $15.2(14.5)$ & $16.3(17.1)$ & $15.2(12.6)$ \\
ADHD IV \%ile & $63.1(28.6)$ & $67.6(30.6)$ & $59.1(27.4)$ \\
F\&V portions & $4.67(1.61)$ & $4.85(1.71)$ & $4.48(1.59)$
\end{tabular}


Thirty minutes before consumption, a confederate mixed active and placebo powders with $30 \mathrm{ml}$ of a low flavonoid orange cordial (Rocks Orange Squash; 8.4 kcal containing $13.2 \mathrm{mg}$ flavonoids in total per $30 \mathrm{ml}$ ) and $170 \mathrm{ml}$ of water into an opaque plastic cup with opaque lid.

Treatments were consumed through a black straw by the participant. The participant and experimenter therefore remained blind to the treatment on each test day.

E-Prime V2 (Psychology Software Tools, Inc.) running on a PC with a 15" screen was used to display the stimuli and record participant responses. Playground noise was played through Sennheiser HD202 headphones at a level of $86 \mathrm{~dB}$.

\section{Task development}

The task is a modification of the Attention Network Task (ANT).$^{27}$ As described above, participants respond to a central arrow which can either be congruent or incongruent to the arrows surrounding it. Additional cues are presented before the stimulus in each trial. From these manipulations, measures of executive attention can be achieved by comparing congruency; measures of alerting can be achieved by comparing trials where cues or no cues are presented; and measures of orienting can be achieved by comparing trials where location or double cues are given. To this we added three further conditions. Response time to target is known to increase as the number of non-target items increases, ${ }^{32}$ therefore a visual load condition was added by varying the number of arrows presented on each trial. There is also a positive relationship between target duration and response time and therefore blocks of short $120 \mathrm{~ms}$ and long $500 \mathrm{~ms}$ targets were employed. Finally, given that the significant findings from the Whyte $e t a l{ }^{21}$ study were found during the school lunch break with an element of playground noise which may have introduced a further element of distraction, a condition with prerecorded playground noise was introduced.

The task was piloted in order to confirm that that load, congruence, cue type and target time effects could be achieved as predicted (see additional material for results).

\section{Modified attention network task (MANT)}

Practice trials. Participants initially completed 36 practice trials. Over the first 18 trials the target duration decreased from $1000 \mathrm{~ms}$ to $120 \mathrm{~ms}$ to facilitate accommodation to the speed of response required. In order to confirm participants could perform the task at above chance levels, if participants scored less than $60 \%$ correct responses on completion of the initial 36 practice trials, further instruction was given followed by additional sets of practice trials. In total 17 participants required additional training, with a single additional practice required by 5 participants, 2, 3, and 5 additional practices were required by 6,5 , and 1 participants, respectively.

Test trials. Participants performed four blocks of the MANT, each consisting of 80 trials. For the main task, participants were initially shown a fixation slide with a cross in the centre of the screen, for $1000 \mathrm{~ms}$. This was followed by either no cue, a centre cue, a double cue or a spatial cue which was displayed

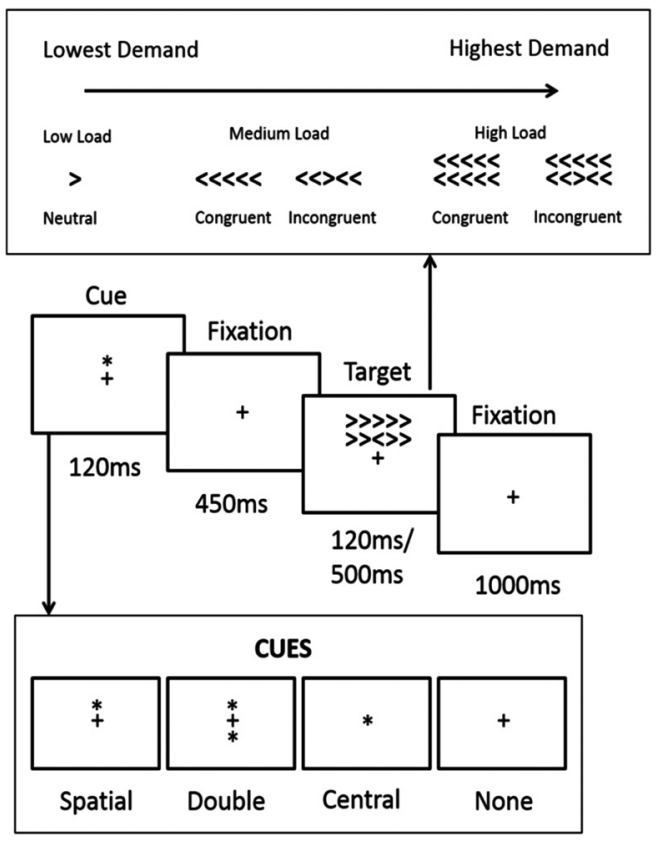

Fig. 1 Schematic of the modified attention network task. Participants completed 4 blocks two which had target duration of $120 \mathrm{~ms}$ and two which had target duration $500 \mathrm{~ms}$. One each of the $120 \mathrm{~ms}$ and $500 \mathrm{~ms}$ blocks were also accompanied by school playground noise. The remaining blocks were accompanied by silence.

for $120 \mathrm{~ms}$ and randomised so that each cue appeared with equiprobability. There was then a further short fixation period of $450 \mathrm{~ms}$. The stimuli were then displayed either above or below the fixation point for $120 \mathrm{~ms}$ (two blocks) or $500 \mathrm{~ms}$ (two blocks) and could be congruent, incongruent or neutral depending on the direction of the arrow and whether there were flanker arrows present (see Fig. 1). Neutral trials were considered to be low visual load with congruent and incongruent trials either being medium visual load (one row of 5 arrows) or high visual load (two rows of 5 arrows). The stimuli position, congruence, and load were randomised so that each was displayed with equiprobability. In addition, one $120 \mathrm{~ms}$ target block and one $500 \mathrm{~ms}$ target block was accompanied by playground noise peaking at no more than $86 \mathrm{~dB}$; other blocks were presented in silence. Participants were instructed to press the mouse button corresponding to the direction the central arrow was facing and had a $1000 \mathrm{~ms}$ response window extending into the following fixation slide. Accuracy and response times for each treatment were measured for congruency, load, target duration and cue type. In order to control for responses made before the stimuli could be properly processed, trials with RTs of less that $100 \mathrm{~ms}$ were removed from the analysis.

\section{Procedure}

Participants were tested on 3 occasions over a 2 weeks period (a baseline session followed by two test sessions). For the 24 hours before each test session, participants were asked to consume a low flavonoid diet. An information sheet containing a list of suitable low flavonoid foods and unsuitable high 
flavonoid foods was given to the parent/legal guardian to facilitate this procedure. In addition, food diaries were completed by the parents or guardians to ensure compliance with the low flavonoid diet for $24 \mathrm{~h}$ preceding each test. Inspection of the food diaries for unsuitable foods confirmed that all participants adhered to the diet. On each test day, the participants consumed a low flavonoid lunch consisting of a ham or cheese sandwich, bag of plain crisps and a banana, provided by the parent/legal guardian. Participants were allowed to consume water ad libitum during each test day. No treatment was given before the baseline/practice session which was always week 1. During weeks 2 and 3 the placebo and WBB treatments were administered in a fully counterbalanced order across participants. There was a seven day wash-out between each treatment. All treatments were consumed at 1300 in the participant's school, $3 \mathrm{~h}$ prior to testing in our laboratories at the University of Reading.

\section{Data treatment}

Given the total number of possible analyses and resulting danger of family-wise error, a number of a priori decisions were taken such that analysis of reaction time and accuracy was restricted to the following:

- A four way drink (2) $\times$ load (2) $\times$ target duration (2) by congruence (2) ANOVA was performed as visual load and response interference are known to interact with duration of target. ${ }^{33,34}$ Given that the single arrow trials count as both low load and neutral congruence stimuli, they were removed from this analysis in order to allow for both load and congruence to be compared together.

- A three way drink (2) $\times$ noise (2) by congruence (3) ANOVA was performed to investigate whether the interference effects found by Whyte et $a .^{21}$ during the lunch time period were mediated by additional distraction from external playground noise.

- The orienting and alerting cognitive networks are believed to operate in isolation to interference effects. ${ }^{27,35}$ It was therefore decided to perform a direct $2 \times 4$ (treatment $\times$ cue) ANOVA analysis as described below.

Where appropriate, following a significant Mauchly's test, the Greenhouse-Geisser correction was applied to compensate for violation of the assumption of sphericity.

As the initial baseline session was treated as a practice session, all analyses were performed on the second two treatment sessions using SPSS V21.

\section{Results}

\section{Analysis of treatment $\times$ target time $\times$ load $\times$ congruence effects}

For the analysis of reaction time, as expected, there were significant main effects for load $\left[F(1,20)=12.2, p=0.002, \eta_{\mathrm{p}}{ }^{2}=\right.$ $0.380]$ and congruence $\left[F(1,20)=59.1, p<0.001, \eta_{\mathrm{p}}{ }^{2}=0.747\right]$ confirming the effect of these manipulations (note, for analyses reported below, the main effects for load and congruence were uniformly significant and are therefore not reported again). Our analysis also revealed a near significant effect of treatment, whereby participants responded faster following blueberry $($ mean $=573 \mathrm{~ms})$ than vehicle $($ mean $=607 \mathrm{~ms})$, $\left[F(1,20)=3.92, p=0.062, \eta_{\mathrm{p}}{ }^{2}=0.164\right]$. Most interestingly, we also saw a significant treatment $\times$ target time $\times$ load interaction, $\left[F(1,20)=5.02, p=0.037, \eta_{\mathrm{p}}{ }^{2}=0.201\right]$.

Data were further split by duration of target and two $2 \times 2 \times 2$ (treatment $\times$ load $\times$ congruence) ANOVAs were performed. No treatment-related main effects or interactions were found for the $120 \mathrm{~ms}$ target duration trials. In contrast, the $500 \mathrm{~ms}$ target time ANOVA revealed a significant main effect of treatment with participants responding faster following blueberry (mean $=566 \mathrm{~ms}$ ) than vehicle (mean $=607 \mathrm{~ms}),[F(1,20)=5.219, p=$ $\left.0.033, \eta_{\mathrm{p}}{ }^{2}=0.207\right]$. Furthermore, there was a significant interaction between drink and load, $\left[F(1,20)=5.6, p=0.028, \eta_{\mathrm{p}}{ }^{2}=\right.$ $0.219]$. All other interactions were non-significant.

To further investigate the interaction between drink and load, the data for the $500 \mathrm{~ms}$ target time trials was split by load and two $2 \times 2$ (treatment $\times$ congruence) ANOVAs were performed. The medium load ANOVA analysis revealed a trend towards significance for the main effect of drink with participants responding faster following blueberry (mean $=565 \mathrm{~ms}$ ) than vehicle $($ mean $=600 \mathrm{~ms}),\left[F(1,20)=3.93, p=0.061, \eta_{\mathrm{p}}{ }^{2}=\right.$ $0.164]$. There was no significant interaction between treatment and congruence, $\left[F(1,20)=3.237, p=0.087, \eta_{\mathrm{p}}{ }^{2}=0.139\right]$. As can be seen in Fig. 2, the high load ANOVA analysis revealed a
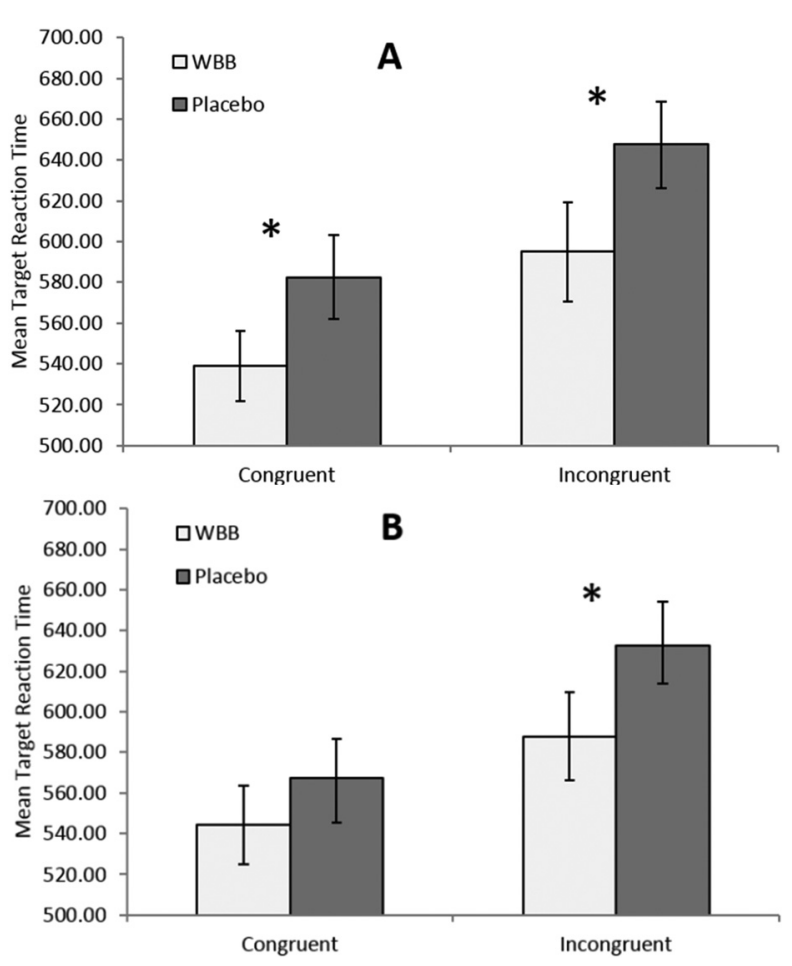

Fig. 2 Mean response times $( \pm 95 \% \mathrm{Cl})$ for $500 \mathrm{~ms}$ targets as a function of treatment, congruence and (A) high visual load and (B) medium visual load. Participants perform significantly faster $(p<0.05)^{*}$ on high visual load/congruent, high visual load/incongruent and medium visual load/ incongruent trials. 
significant effect of treatment, with participants responding more quickly following blueberry (mean $=567 \mathrm{~ms}$ ) than vehicle $($ mean $=614 \mathrm{~ms}),\left[F(1,20)=6.26, p=0.021, \eta_{\mathrm{p}}{ }^{2}=0.238\right]$. There was no significant interaction between these two measures, $\left[F(1,20)=0.432, p=0.518, \eta_{\mathrm{p}}{ }^{2}=0.021\right]$. However, paired samples $t$-test simple main effects analysis further revealed significant differences between treatments where target duration was $500 \mathrm{~ms}$, showing a significant difference between treatments for high load congruent trials, $[t(20)=-2.4, p=0.026$, $d=-0.53]$, high load incongruent trials, $[t(20)=-2.35, p=$ $0.029, d=-0.53]$, and medium load incongruent trials, $[t(20)=$ $-2.5, p=0.021, d=-0.55]$. As hypothesised, it would seem that the effect of the blueberry treatment becomes most marked during the more cognitively demanding trials, where presentation rate is $500 \mathrm{~ms}$, and where trials are incongruent and have a high load, but not where trials are congruent with a medium load.

For accuracy, as expected, there were main effects for load $\left[F(1,20)=5.59, p=0.028, \eta_{\mathrm{p}}{ }^{2}=0.218\right]$ and congruence $[F(1,20)$ $\left.=40.4, p<0.001, \eta_{\mathrm{p}}{ }^{2}=0.669\right]$. A significant target time $\times$ load interaction was also found $\left[F(1,20)=7.13, p=0.015, \eta_{\mathrm{p}}{ }^{2}=\right.$ 0.263] along with trends towards significance for the treatment $\times$ load $\left[F(1,20)=3.70, p=0.069, \eta_{\mathrm{p}}{ }^{2}=0.156\right]$ and treatment $\times$ target time $\times$ load $\left[F(1,20)=3.47, p=0.077, \eta_{\mathrm{p}}{ }^{2}=\right.$ 0.148]. Data were further split by load, however, beyond the expected main effect of congruence, all other main effects and interactions were found to be non-significant.

\section{Analysis of treatment $\times$ noise $\times$ congruence effects}

The three way $2 \times 2 \times 3$ (treatment $\times$ noise $\times$ congruence) ANOVA revealed a significant main effect for treatment whereby participants responded faster following $\mathrm{WBB}$ (mean = $563 \mathrm{~ms}$ ) than vehicle (mean $=597 \mathrm{~ms}),[F(1,20)=4.43, p=$ $\left.0.048, \eta_{\mathrm{p}}{ }^{2}=0.181\right]$. A significant main effect was also found for noise whereby participants responded more slowly in silence (mean $586 \mathrm{~ms}$ ) than with playground noise (mean $=573 \mathrm{~ms}$ ), $\left[F(1,20)=7.8, p=0.011, \eta_{\mathrm{p}}{ }^{2}=0.218\right]$. As shown in Fig. 3 , there was a significant main effect of congruence whereby participants responded more slowly for incongruent trials $($ mean $=$ $619 \mathrm{~ms})$ than congruent trials $($ mean $=561)$ and neutral trials $($ mean $=559 \mathrm{~ms}),\left[F(2,40)=50.3, p<0.001, \eta_{\mathrm{p}}{ }^{2}=0.715\right]$. No significant interactions were found for this analysis. Beyond the expected effect of congruence, $\left[F(2,40)=22.3, p<0.001, \eta_{\mathrm{p}}{ }^{2}=\right.$ 0.527 ], no treatment related significant effects were found for the accuracy measures of this analysis.

\section{Effect of Cue type on performance}

The treatment (2) $\times$ cue (4) ANOVA analysis of reaction time revealed a main effect trend of treatment, with participants responding more rapidly following blueberry $($ mean $=566 \mathrm{~ms})$ than vehicle $($ mean $=601 \mathrm{~ms}),\left[F(1,20)=4.31, p=0.51, \eta_{\mathrm{p}}{ }^{2}=\right.$ $0.177]$. There was also a significant main effect of cue with participants most notably responding more slowly on non-cue trials $($ mean $=611 \mathrm{~ms}$ ) in comparison to central (mean $=$ $579 \mathrm{~ms})$, double $($ mean $=575 \mathrm{~ms})$, and spatial $($ mean $=568 \mathrm{~ms})$ cues, $\left[F(3,60)=33.5, p<0.001, \eta_{\mathrm{p}}{ }^{2}=0.626\right]$. The interaction

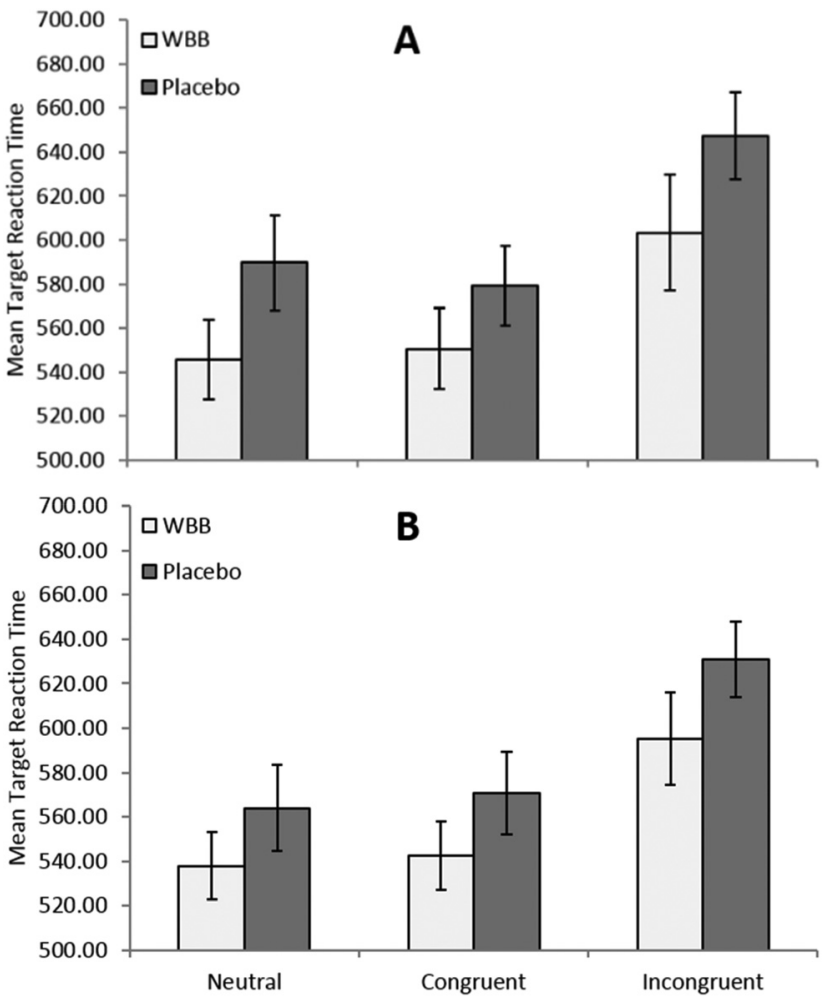

Fig. 3 Mean response times $( \pm 95 \% \mathrm{Cl})$ as a function of treatment, congruence and (A) silence and (B) noise. Participants respond more rapidly following vehicle in the noise condition when compared to the silent condition. There is less change in performance between noise and silent conditions for the WBB interventions.

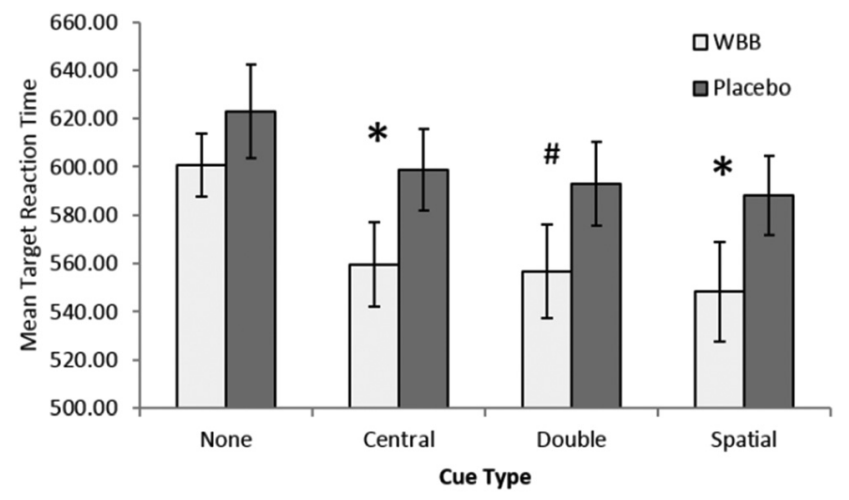

Fig. 4 Mean response times $( \pm 95 \% \mathrm{Cl})$ as a function of treatment and cue type. Response times are significantly $(<0.05)^{*}$ faster following WBB for both central and spatial cues, there is also a trend $(<0.10) \#$ for double cues, however, there is less effect where no cue is presented.

between treatment and cue also showed a trend towards significance, $\left[F(3,60)=2.66, p=0.056, \eta_{\mathrm{p}}{ }^{2}=0.117\right]$.

Given the near significant interaction, a simple main effects analysis was performed comparing treatment for each cue type using paired samples $t$-tests. As can be seen in Fig. 4 , this analysis further revealed a significant central cue effect, $[t(20)=-2.28, p=0.033, d=-0.5]$, a significant spatial cue 
effect, $[t(20)=-2.189, p=0.041, d=-0.48]$, and double cue trend, $[t(20)=-2, p=0.059, d=-0.44]$. No effect was found on the no cue trials, $[t(20)=-1.5, p=0.150, d=-0.32]$.

Taken as whole these findings indicate that, regardless of type, the presentation of a cue before target presentation facilitates faster response times following blueberry treatment when compared with vehicle.

No significant effects were found for the treatment $(2) \times$ cue (4) ANOVA accuracy analysis.

\section{Speed - accuracy trade off}

As reported above, there was no significant difference between treatments for the measures of accuracy, indicating that the WBB related improvements in reaction time were not achieved with a cost to accuracy. One participant was found to be performing at chance for accuracy in both conditions $(\mathrm{WBB}=0.049$, placebo $=0.047)$ and one for WBB only $(0.42)$. The relationship between speed and accuracy was investigated further by calculating each participant's inverse efficiency scores, dividing overall proportion correct responses by mean reaction time for each treatment. There was no significant difference between treatments $[t(20)=$ $-0.751, p=0.462]$.

\section{Discussion}

Previously, flavonoid research involving children has primarily shown positive effects of intervention within the domain of memory performance with only one study indicating a possible accuracy benefit on the incongruent trails of a response interference task. ${ }^{21,22,36}$ Evidence for beneficial effects on executive function following an acute flavonoid rich intervention within this age group were therefore equivocal though it was hypothesised that this was a function of previous tasks lacking sensitivity. Here, for the first time, we have demonstrated significantly faster response times following a wild blueberry intervention on a task of executive function and highlighted the conditions under which these effects might be found. Analysis revealed that, as hypothesised, the benefits of the blueberry treatment were evident on more cognitively demanding high load or incongruent trials indicating a beneficial effect on EF under these conditions. These effects were particularly evident where stimulus presentation was $500 \mathrm{~ms}$. Though it had been hypothesised that participants would perform more accurately following blueberry treatment on incongruent/noise trials, this effect proved to be non-significant, with noise having no effect on accuracy performance. No speed-accuracy trade-off was evident, though it should be noted that two participants did perform at chance. Finally, given the overarching hypothesis that, as cognitive demand increased, the benefits of WBB treatment would increase in comparison to vehicle, one might have expected to find the greatest difference between treatments to be found on the non-cue trials. This was not the case, with a significant advantage for WBB treatment being found for each type of cued trial.
Our study was specifically designed to test the hypothesis that as cognitive demand increases, a flavonoid driven advantage will emerge whereby the difference in performance between WBB and vehicle treatments will increase up to the point where the task becomes overly demanding for effective performance to continue. This idea was not fully supported, with some elements (such as cueing) seemingly facilitating better WBB-related performance irrespective of task difficulty. Nonetheless, we observed a cognitive load and congruence effect with participants responding more rapidly following WBB intervention in the more cognitively demanding high load and incongruent conditions where target duration was slower. Furthermore, as Simon and Berbaum ${ }^{34}$ report on a response interference Stroop task, increasing stimulus duration led to slower response times. Welford ${ }^{37}$ proposes that the time taken to integrate information before making a response increases in line with stimulus duration. Where duration is short, as with the $120 \mathrm{~ms}$ duration, at stimulus offset no further information is available at the point the response is made. The longer $500 \mathrm{~ms}$ duration extends the period for stimulus sampling and integration. This increases processing of the interference elements of the stimulus. Better performance on the incongruent and high load trials at $500 \mathrm{~ms}$ but not at $120 \mathrm{~ms}$ can therefore be argued to demonstrate a WBB benefit-i.e. overcoming the effects of these cognitively demanding elements when they are more challenging.

It had been considered that the playground noise present during the lunch time session in our previous study may have proven distracting and therefore increased cognitive load. We therefore hypothesised that by introducing such noise in the current study better performance might be found following WBB in comparison to placebo. This was not the case, with no effect between treatments being found for this condition. One possible explanation for the lack of effect found here is that the sound file had been compressed to give a dynamic range of no more than $20 \mathrm{~dB}$. Reducing the dynamic range in this way may have made the noise more constant in nature and reduced the startling nature of any sudden changes in level. Previous research has found that constant sound, such as white noise, can have a positive effect on cognitive performance on executive function tasks in 8-10 years old children dependent on their attentional capabilities ${ }^{38}$ and it is possible that the noise used in the current study may have mediated performance in such a way as to mask any positive WBB effects. It should be noted that exploratory analysis including ADHD IV (as a proxy for attentional capabilities) ratings as a covariate in a treatment by noise ANOVA (not reported here) found no significant effects, however, future research should consider a noise distractor with a greater dynamic range and more distracting nature.

No physiological measures were taken during this study however, studies considering the acute effects of flavonoid interventions on cerebral blood flow (CBF) with young adults have shown increased perfusion in areas such as the middle frontal gyrus, pre-central gyrus and occipital cortex following acute anthocyanin rich blueberry treatment ${ }^{18}$ with older adults 
showing increased perfusion in the anterior cingulate cortex (ACC) and central opercular cortex of the parietal lobe following acute cocoa flavanol treatment. ${ }^{39}$ Of particular interest here is the increased CBF in the frontal, particularly dorsolateral prefrontal (DLPFC), and ACC areas. These areas are believed to be important for detecting and resolving interference in Stroop and Flanker tasks similar to the one used in the current study. ${ }^{40}$ It could therefore be posited that a possible mechanism underlying the improved executive function performance found in the current study is the facilitation of performance by increased blood flow to key areas required to effectively perform our response interference MANT task. Additionally, fMRI studies have found that, following chronic blueberry interventions, aging adults have shown increased BOLD activation of the ACC and frontal areas during performance of an n-back task, ${ }^{17}$ and also the left middle frontal gyrus during a Stroop task. ${ }^{25}$ Interestingly the increased activation did not lead to improved performance for the Stroop, a similar executive function response interference task to the MANT, however in a recent chronic blueberry intervention with aging adults Miller et $a .^{26}$ found improvements in executive function tasks which were primarily reliant on the frontal region of the brain and were also considered to be the most challenging in the battery employed. These findings give further credence to the proposal that, even though a cognitive domain, such as executive function, may be responsive to flavonoid intervention and physiological effects may be apparent, the intervention may not show beneficial behavioural changes unless the task is sufficiently sensitive as found by Miller et al. ${ }^{26}$ and also in our current study.

The implications of the cueing effect found here are interesting given they suggest that, in addition to the positive executive function-related effect, there would also seem to be a positive WBB-related alerting network effect. The ACC and DLPFC which, as discussed above, show increased activation in cognitive tasks following blueberry anthocyanin intervention have been found to be important for phasic alertness ${ }^{41-43}$ and it is therefore proposed that this previous research is consistent with our current findings.

An alternative interpretation of the results here is that the comparative increase in complexity and demand inherent in the current task may have facilitated the global improvement in treatment related performance rather than a particular combination of cognitively demanding conditions. Scholey et al. ${ }^{16}$ propose that in addition to task sensitivity, sustained cognitive effort is required to reveal differences in flavonoid related performance, and indeed their research findings would seem to support this. The findings here indicate that, by increasing the overall level of cognitive demand of the task, the need for longer periods of sustained cognitive effort to show an effect is lessened. Further research of the MANT with an adult sample would therefore be recommended to investigate whether this effect might extend to an adult population.

Our current findings add to previous research with primary school age children which have shown improved memory function following flavonoid related interventions. Taken together with the clear improvements in executive function perform- ance shown here, it can be proposed that supplementation with WBB would be beneficial in a learning environment to boost both memory and attention. Indeed, there is good reason to posit that intervention with WBB may lead to direct improvements in the classroom. Executive function abilities are believed to be crucial to academic performance. Performance on tests of working memory, and inhibition (similar to the MANT) have been found to be predictive of maths and reading ability in elementary school aged children. ${ }^{44-46}$ Additionally, Rueda et $a .^{47}$ found that, following 5 days of attention training, children 4 and 6 years of age improved in performance on the Attention Network Task (from which the MANT was developed) and, importantly, this improvement in executive function ability also transferred to improved fluid intelligence. It is therefore possible that our WBB related improvements in executive function may transfer to IQ and academic performance. Further support for the impact of $\mathrm{WBB}$ on academic achievement comes from the finding that significant improvements in reaction time of up to $9 \%$ were found in comparison to placebo. This indicates an improvement in information processing speed. Importantly, positive significant relationships have previously been found between processing speed and academic achievement in areas such as maths, reading, and language skills ${ }^{48-53}$ and in a review of the intelligence and speed of information processing literature, Sheppard and Vernon ${ }^{54}$ found that measures of intelligence were also significantly related to processing speed. To our knowledge, at present no studies have been published considering the effect of a flavonoid intervention on academic outcomes in children, however, given the relationship between executive function and processing speed found in the current study, further research is recommended to consider the chronic effects of WBB intervention on these measures. Furthermore, the positive effect on cognitive function found here is also important in relation to long-term outcomes. Cognitive function in older adults is substantially determined by peak cognitive ability attained in childhood, ${ }^{55}$ and poorer cognitive function in childhood is a risk factor for common mental health problems many years later. ${ }^{56}$ Thus, continued WBB supplementation within this age group could have a significant impact on lifelong cognitive and mental health.

\section{Conclusions}

The results of this study suggest that positive effects of blueberry flavonoid intervention can be found within the domain of executive function for younger children. This effect was most evident on incongruent and high load trials, suggesting performance can be maintained on the more cognitively demanding aspects of the task following WBB treatment when compared with placebo. Conversely, a cueing effect was found where WBB positively affected phasic alertness indicating that a higher level of cognitive demand may not always be necessary for a difference to be shown. A further interpretation of the results, therefore, is that rather than any effect of individual 
variables, the increased cognitive demand of the task as whole may be the most important factor for a difference in performance between treatment conditions to be found.

\section{Conflicts of interest}

There are no conflicts of interest to declare.

\section{Acknowledgements}

This research was funded by a University of Reading Social Sciences doctoral studentship to A. W. We are grateful to the Wild Blueberry Association of North America who provided the freeze dried blueberries used in this study.

\section{Notes and references}

1 S. Desch, J. Schmidt, D. Kobler, M. Sonnabend, I. Eitel, M. Sareban, K. Rahimi, G. Schuler and H. Thiele, Effect of cocoa products on blood pressure: systematic review and meta-analysis, Am. J. Hypertens., 2010, 23, 97-103.

2 M. G. Shrime, S. R. Bauer, A. C. McDonald, N. H. Chowdhury, C. E. Coltart and E. L. Ding, Flavonoidrich cocoa consumption affects multiple cardiovascular risk factors in a meta-analysis of short-term studies, J. Nutr., 2011, 141, 1982-1988.

3 L. Bell, D. J. Lamport, L. T. Butler and C. M. Williams, A review of the cognitive effects observed in humans following acute supplementation with flavonoids, and their associated mechanisms of action, Nutrients, 2015, 7, 10290-10306.

4 D. J. Lamport, L. Dye, J. D. Wightman and C. L. Lawton, The effects of flavonoid and other polyphenol consumption on cognitive performance: a systematic research review of human experimental and epidemiological studies, Nutr. Aging, 2012, 1, 5-25.

5 A. L. Macready, O. B. Kennedy, J. A. Ellis, C. M. Williams, J. P. Spencer and L. T. Butler, Flavonoids and cognitive function: a review of human randomized controlled trial studies and recommendations for future studies, Genes Nutr., 2009, 4, 227-242.

6 J. A. Joseph, B. Shukitt-Hale, N. A. Denisova, D. Bielinski, A. Martin, J. J. McEwen and P. C. Bickford, Reversals of agerelated declines in neuronal signal transduction, cognitive, and motor behavioral deficits with blueberry, spinach, or strawberry dietary supplementation, J. Neurosci., 1999, 19, 8114-8121.

7 M. G. Miller and B. Shukitt-Hale, Berry fruit enhances beneficial signaling in the brain, J. Agric. Food Chem., 2012, 60, 5709-5715.

8 C. Rendeiro, D. Vauzour, M. Rattray, P. Waffo-Téguo, J. M. Mérillon, L. T. Butler, C. M. Williams and J. P. Spencer, Dietary levels of pure flavonoids improve spatial memory performance and increase hippocampal brain-derived neurotrophic factor, PLoS One, 2013, 8, e63535.

9 N. Thangthaeng, S. M. Poulose, S. M. Gomes, M. G. Miller, D. F. Bielinski and B. Shukitt-Hale, Tart cherry supplementation improves working memory, hippocampal inflammation, and autophagy in aged rats, Age, 2016, 38, 393404.

10 C. M. Williams, M. A. El Mohsen, D. Vauzour, C. Rendeiro, L. T. Butler, J. A. Ellis, M. Whiteman and J. P. Spencer, Blueberry-induced changes in spatial working memory correlate with changes in hippocampal CREB phosphorylation and brain-derived neurotrophic factor (BDNF) levels, Free Radical Biol. Med., 2008, 45, 295-305.

11 R. J. Kean, D. J. Lamport, G. F. Dodd, J. E. Freeman, C. M. Williams, J. A. Ellis, L. T. Butler and J. P. Spencer, Chronic consumption of flavanone-rich orange juice is associated with cognitive benefits: an $8-w k$, randomized, double-blind, placebo-controlled trial in healthy older adults, Am. J. Clin. Nutr., 2015, 101, 506-514.

12 M. H. Alharbi, D. J. Lamport, G. F. Dodd, C. Saunders, L. Harkness, L. T. Butler and J. P. Spencer, Flavonoid-rich orange juice is associated with acute improvements in cognitive function in healthy middle-aged males, Eur. J. Nutr., 2016, 55, 2021-2029.

13 A. W. Watson, C. F. Haskell-Ramsay, D. O. Kennedy, J. M. Cooney, T. Trower and A. Scheepens, Acute supplementation with blackcurrant extracts modulates cognitive functioning and inhibits monoamine oxidase-B in healthy young adults, J. Funct. Foods, 2015, 17, 524-539.

14 S. Elsabagh, D. E. Hartley, O. Ali, E. M. Williamson and S. E. File, Differential cognitive effects of Ginkgo biloba after acute and chronic treatment in healthy young volunteers, Psychopharmacology, 2005, 179, 437-446.

15 D. O. Kennedy, A. B. Scholey and K. A. Wesnes, The dosedependent cognitive effects of acute administration of Ginkgo biloba to healthy young volunteers, Psychopharmacology, 2000, 151, 416-423.

16 A. B. Scholey, S. J. French, P. J. Morris, D. O. Kennedy, A. L. Milne and C. F. Haskell, Consumption of cocoa flavanols results in acute improvements in mood and cognitive performance during sustained mental effort, J. Psychopharmacol., 2010, 24, 1505-1514.

17 J. L. Bowtell, Z. Aboo-Bakkar, M. Conway, A.-L. R. Adlam and J. Fulford, Enhanced task related brain activation and resting perfusion in healthy older adults after chronic blueberry supplementation, Appl. Physiol., Nutr., Metab., 2017, 773-779.

18 G. F. Dodd, The acute effects of flavonoid-rich blueberries on cognitive function in healthy younger and older adults, University of Reading, 2012.

19 D. T. Field, C. M. Williams and L. T. Butler, Consumption of cocoa flavanols results in an acute improvement in visual and cognitive functions, Physiol. Behav., 2011, 103, 255-260.

20 D. J. Lamport, D. Pal, A. L. Macready, S. Barbosa-Boucas, J. M. Fletcher, C. M. Williams, J. P. Spencer and L. T. Butler, The effects of flavanone-rich citrus juice on 
cognitive function and cerebral blood flow: an acute, randomised, placebo-controlled cross-over trial in healthy, young adults, Br. J. Nutr., 2016, 116, 2160-2168.

21 A. R. Whyte, G. Schafer and C. M. Williams, Cognitive effects following acute wild blueberry supplementation in 7-to 10-year-old children, Eur. J. Nutr., 2016, 55, 2151-2162.

22 A. R. Whyte and C. M. Williams, Effects of a single dose of a flavonoid-rich blueberry drink on memory in 8 to $10 \mathrm{y}$ old children, Nutrition, 2015, 31, 531-534.

23 D. Camfield, A. Scholey, A. Pipingas, R. Silberstein, M. Kras, K. Nolidin, K. Wesnes, M. Pase and C. Stough, Steady state visually evoked potential (SSVEP) topography changes associated with cocoa flavanol consumption, Physiol. Behav., 2012, 105, 948-957.

24 S. Francis, K. Head, P. Morris and I. Macdonald, The effect of flavanol-rich cocoa on the fMRI response to a cognitive task in healthy young people, J. Cardiovasc. Pharmacol., 2006, 47, S215-S220.

25 E. L. Boespflug, J. C. Eliassen, J. A. Dudley, M. D. Shidler, W. Kalt, S. S. Summer, A. L. Stein, A. N. Stover and R. Krikorian, Enhanced neural activation with blueberry supplementation in mild cognitive impairment, Nutr. Neurosci., 2017, 1-9.

26 M. G. Miller, D. A. Hamilton, J. A. Joseph and B. ShukittHale, Dietary blueberry improves cognition among older adults in a randomized, double-blind, placebo-controlled trial, Eur. J. Nutr., 2017, 1-12.

27 J. Fan, B. D. McCandliss, T. Sommer, A. Raz and M. I. Posner, Testing the efficiency and independence of attentional networks, J. Cogn. Neurosci., 2002, 14, 340-347.

28 J. C. Raven and J. H. Court, Raven's progressive matrices and vocabulary scales, Oxford Psychologists Press Oxford, UK, 1998.

29 G. Dupaul, T. Power, A. Anastopoulos and R. Reid, ADHD rating scale-IV, Guilford, New York, NY, USA, 1998.

30 R. C. Oldfield, The assessment and analysis of handedness: the Edinburgh inventory, Neuropsychologia, 1971, 9, 97-113.

31 A. Rodriguez-Mateos, T. Cifuentes-Gomez, S. Tabatabaee, C. Lecras and J. P. Spencer, Procyanidin, anthocyanin, and chlorogenic acid contents of highbush and lowbush blueberries, J. Agric. Food Chem., 2012, 60, 5772-5778.

32 G. Murphy, J. A. Groeger and C. M. Greene, Twenty years of load theory-Where are we now, and where should we go next?, Psychon. Bull. Rev., 2016, 23, 1316-1340.

33 G. A. Alvarez and P. Cavanagh, The capacity of visual shortterm memory is set both by visual information load and by number of objects, Psychol. Sci., 2004, 15, 106-111.

34 J. R. Simon and K. Berbaum, Effect of conflicting cues on information processing: the 'Stroop effect'vs. the 'Simon effect', Acta Psychol., 1990, 73, 159-170.

35 M. R. Rueda, J. Fan, B. D. McCandliss, J. D. Halparin, D. B. Gruber, L. P. Lercari and M. I. Posner, Development of attentional networks in childhood, Neuropsychologia, 2004, 42, 1029-1040.

36 L. Calderón-Garcidueñas, A. Mora-Tiscareño, M. FrancoLira, J. V. Cross, R. Engle, M. Aragón-Flores, G. Gómez-
Garza, V. Jewells, L. Weili and H. Medina-Cortina, Flavonolrich dark cocoa significantly decreases plasma endothelin1 and improves cognition in urban children, Front. Pharmacol., 2013, 4, 104.

37 A. T. Welford, Reaction times, Academic Pr, 1980.

38 S. K. Helps, S. Bamford, E. J. Sonuga-Barke and G. B. Söderlund, Different effects of adding white noise on cognitive performance of sub-, normal and super-attentive school children, PLoS One, 2014, 9, e112768.

39 D. J. Lamport, D. Pal, C. Moutsiana, D. T. Field, C. M. Williams, J. P. Spencer and L. T. Butler, The effect of flavanol-rich cocoa on cerebral perfusion in healthy older adults during conscious resting state: a placebo controlled, crossover, acute trial, Psychopharmacology, 2015, 232, 32273234.

40 D. E. Nee, T. D. Wager and J. Jonides, Interference resolution: insights from a meta-analysis of neuroimaging tasks, Cogn., Affect., Behav. Neurosci., 2007, 7, 1-17.

41 D. Mannarelli, C. Pauletti, A. Grippo, A. Amantini, V. Augugliaro, A. Currà, P. Missori, N. Locuratolo, M. C. De Lucia and S. Rinalduzzi, The role of the right dorsolateral prefrontal cortex in phasic alertness: evidence from a contingent negative variation and repetitive transcranial magnetic stimulation study, Neural Plast., 2015, 2015, 410785.

42 B. Périn, O. Godefroy, S. Fall and G. De Marco, Alertness in young healthy subjects: an fMRI study of brain region interactivity enhanced by a warning signal, Brain Cogn., 2010, 72, 271-281.

43 M. K. Rothbart and M. I. Posner, The developing brain in a multitasking world, Dev. Rev., 2015, 35, 42-63.

44 J. R. Best, P. H. Miller and L. L. Jones, Executive functions after age 5: Changes and correlates, Dev. Rev., 2009, 29, 180-200.

45 E. H. Gerst, P. T. Cirino, J. M. Fletcher and H. Yoshida, Cognitive and behavioral rating measures of executive function as predictors of academic outcomes in children, Child Neuropsychol., 2017, 23, 381-407.

46 H. L. St Clair-Thompson and S. E. Gathercole, Executive functions and achievements in school: Shifting, updating, inhibition, and working memory, Q. J. Exp. Psychol., 2006, 59, 745-759.

47 M. R. Rueda, M. K. Rothbart, B. D. McCandliss, L. Saccomanno and M. I. Posner, Training, maturation, and genetic influences on the development of executive attention, Proc. Natl. Acad. Sci. U. S. A., 2005, 102, 1493114936.

48 R. G. Floyd, J. J. Evans and K. S. McGREW, Relations between measures of Cattell-Horn-Carroll (CHC) cognitive abilities and mathematics achievement across the schoolage years, Psychol. Sch., 2003, 40, 155-171.

49 D. C. Geary, Cognitive predictors of achievement growth in mathematics: a 5-year longitudinal study, Dev. Psychol., 2011, 47, 1539.

50 T. E. Rohde and L. A. Thompson, Predicting academic achievement with cognitive ability, Intelligence, 2007, 35, 83-92. 
51 Y. A. Dodonova and Y. S. Dodonov, Processing speed and intelligence as predictors of school achievement: Mediation or unique contribution?, Intelligence, 2012, 40, 163-171.

52 J. J. Evans, R. G. Floyd, K. S. McGrew and M. H. Leforgee, The relations between measures of Cattell-Horn-Carroll (CHC) cognitive abilities and reading achievement during childhood and adolescence, School Psychol. Rev., 2002, 31, 246.

53 D. Luo, L. A. Thompson and D. K. Detterman, The criterion validity of tasks of basic cognitive processes, Intelligence, 2006, 34, 79-120.
54 L. D. Sheppard and P. A. Vernon, Intelligence and speed of information-processing: A review of 50 years of research, Pers. Individ. Diff., 2008, 44, 535-551.

55 A. J. Gow, W. Johnson, A. Pattie, C. E. Brett, B. Roberts, J. M. Starr and I. J. Deary, Stability and change in intelligence from age 11 to ages 70, 79, and 87: the Lothian Birth Cohorts of 1921 and 1936, Psychol. Aging, 2011, 26, 232.

56 S. L. Hatch, P. B. Jones, D. Kuh, R. Hardy, M. E. Wadsworth and M. Richards, Childhood cognitive ability and adult mental health in the British 1946 birth cohort, Social Sci. Med., 2007, 64, 2285-2296. 